\title{
Bioinformatic and experimental analyses of key biomarkers in pancreatic cancer
}

\author{
TIANYU REN $^{1 *}$, XIAOFEI XUE ${ }^{2 *}$, XIAOGANG WANG ${ }^{2 *}$, XINGTONG ZHOU ${ }^{1}$ and SHENGCHUN DANG ${ }^{1,2}$ \\ ${ }^{1}$ Department of General Surgery, The Affiliated Hospital, Jiangsu University, Zhenjiang, Jiangsu 212001; \\ ${ }^{2}$ Department of General Surgery, Pucheng Hospital, Weinan, Shaanxi 715500, P.R. China
}

Received February 29, 2020; Accepted September 15, 2020

DOI: $10.3892 /$ etm.2021.10794

\begin{abstract}
The present study aimed to screen the key genes in pancreatic cancer and to explore the pathogenesis of pancreatic cancer. A total of three expression profiling datasets (GSE28735, GSE16515 and GSE15471) associated with pancreatic cancer were retrieved from the public gene chip database. The differentially expressed genes (DEGs) were screened by GEO2R and subjected to Gene Ontology (GO) and signaling pathway enrichment analysis. Furthermore, a protein interaction network was constructed. The GEPIA online database was used to screen for genes that affect the prognosis of pancreatic cancer. Finally, cell functional experiments were performed on the selected key genes. A total of 72 DEGs were identified, including 52 upregulated and 20 downregulated genes. Enrichment analysis revealed roles of the DEGs in endodermal cell differentiation, cell adhesion, extracellular matrix-receptor interaction and PI3K-Akt signaling pathway. In total, 10 key nodal genes were identified, including integrin subunit $\alpha 2$ (ITGA2), ITGB6 and collagen $\alpha 1$ chain 1 . Through survival analysis, two genes with an impact on the prognosis of pancreatic cancer were identified, namely ITGA2 and ITGB6. Silencing of ITGB6 in a pancreatic cancer cell line significantly suppressed cell proliferation and induced
\end{abstract}

Correspondence to: Professor Shengchun Dang, Department of General Surgery, The Affiliated Hospital, Jiangsu University, 438 The Liberation of North Road, Zhenjiang, Jiangsu 212001, P.R. China

E-mail: dscgu@163.com

${ }^{*}$ Contributed equally

Abbreviations: DEG, differentially expressed gene; GEO, Gene Expression Omnibus; GO, Gene Ontology; KEGG, Kyoto Encyclopedia of Genes and Genomes; PPI, protein-protein interaction; STRING, Search Tool for the Retrieval of Interacting Genes/proteins; FN1, fibronectin 1; ITGA2, integrin subunit $\alpha$ 2; COL1A1, collagen $\alpha 1$ chain 1; EMT, epithelial-mesenchymal transition; PSCs, pancreatic stellate cells

Key words: pancreatic cancer, bioinformatics, hub genes, prognosis, integrin subunit $\beta 6$ cell cycle arrest at G2/M phase. The identified key genes and signaling pathways may help to deepen the understanding of the molecular mechanisms involved in pancreatic cancer and provide a theoretical basis to develop novel therapies.

\section{Introduction}

Pancreatic adenocarcinoma (PAAD) is a gastrointestinal malignancy with high mortality. The 5-year survival rate for patients with pancreatic cancer is $<5 \%(1,2)$. It is expected that pancreatic cancer will rank second in terms of mortality rate among cancers worldwide in the next 20 years (3). For patients with pancreatic cancer, the only cure is surgical resection, but the majority of patients are diagnosed with local inoperable tumors or distant metastasis; thus, patients with pancreatic cancer have a poor prognosis $(4,5)$. Therefore, further research is urgently required to develop effective prevention measures and early diagnostic methods. In the past few decades, efforts to study the molecular mechanisms of pancreatic cancer have provided hope for molecular diagnostics and molecular targeted therapy for various diseases.

Genes with significantly high expression levels in pancreatic cancer include KRAS, BRAF serine/threonine kinase (BRAF) and AKT serine/threonine kinase 2 (AKT2), as previously reported (6). These genes may be used as biomarkers for early diagnosis of pancreatic cancer. Gene expression profile analysis is a high-throughput method for detecting mRNA expression in tissues or cell samples. For instance, based on data from Gene Expression Omnibus (GEO), Long et al (7) screened the differentially expressed genes (DEGs) in pancreatic cancer and analyzed the copy number variation in DEGs. Their study indicated that transforming growth factor $\beta$ receptor 1 (TGFBR1) and transforming growth factor $\beta 1$ (TGFB1) have an important role in the development of pancreatic cancer. The Wnt (8) and hedgehog (9) signaling pathways have been identified as being of marked significance in pancreatic cancer. microRNAs (miRNAs or miRs) have attracted widespread attention in recent years (10). A previous study indicated that miRNA-27a promotes the proliferation of pancreatic cancer cells by activating the Wnt/ $\beta$-catenin signaling pathway (11). miR-132 has recently been demonstrated to promote pancreatic cancer cell proliferation and inhibit apoptosis through the hedgehog signaling pathway (12). Despite these tremendous advances, the underlying key mechanisms of pancreatic cancer 
require to be further elucidated to screen promising prognostic biomarkers and potential targets for diagnosis and treatment of pancreatic cancer. In the present study, genes associated with pancreatic cancer were determined from datasets obtained from the online database GEO.GEO2R analysis was performed to identify the DEGs associated with pancreatic cancer (13). Further enrichment analysis of DEGs was applied to explore the molecular mechanisms associated with pancreatic cancer. The core genes in the development of pancreatic cancer were then explored through the analysis of differential gene-protein networks and sub-network modules. Overexpression of integrin subunit $\alpha 2$ (ITGA2) and ITGB6 was determined to be associated with poor prognosis. Silencing of ITGB6 inhibited cell proliferation in pancreatic cancer and produced cell cycle arrest at $\mathrm{G} 2 / \mathrm{M}$ phase.

\section{Materials and methods}

Screening of DEGs from GEO datasets. The GEO database is an international public database of datasets, including data from single- and dual-channel determination of mRNA expression and experimental data for genomic DNA and proteins (14). In the present study, three expression profiling datasets [GSE28735 (15), GSE16515 (16) and GSE15471 (17)] were downloaded from GEO (https://www.ncbi.nlm.nih. gov/geo/). GSE15471 contains 39 tumor and paired adjacent normal tissues; GSE16515 contains 36 tumor and 16 adjacent normal tissues; and GSE28735 contains 45 tumor and paired adjacent normal tissues. GEO2R (13) was used to screen for DEGs, and those DEGs shared by the three sets of expression profiles were further selected by the Venn mapping tool. A $\log 2$ fold change $>1.5$ and adjusted $\mathrm{P}<0.05$ were considered to indicate a statistically significant difference.

Database for Annotation, Visualization and Integrated Discovery database enrichment analysis. Cluster Profiler is an ontology-based $\mathrm{R}$ package that automates the process of biological-term classification and the enrichment analysis of gene clusters, and provides a visualization module for displaying the analysis results (18). DEGs were subjected to Gene Ontology (GO) enrichment analysis [molecular function (MF), biological process (BP) and cellular component $(19,20)]$ and signaling pathway Kyoto Encyclopedia of Genes and Genomes (KEGG) enrichment analysis using Cluster Profiler V3.6.0. The enrichment analysis and function annotation data were obtained and displayed in the form of a bubble chart. $\mathrm{P}<0.05$ was considered to indicate a statistically significant difference.

Protein interaction network construction and sub-network module analysis. The Search Tool for the Retrieval of Interacting Genes/proteins (STRING; https://string-db.org/) is an online tool for searching for gene interactions and protein interactions (21). Selected DEGs were inputted into the online database STRING to generate a protein network diagram, which was visualized by Cytoscape v3.7.0 software (https://cytoscape.org/). Degree was used as the criterion for screening key target genes (where the degree of nodes indicates the number of proteins that the nodes are able to interact with). The sub-network modules related to pancreatic cancer development were analyzed using MCODE plug-in.
Gene Expression Profiling Interactive Analysis (GEPIA) online survival analysis. GEPIA is a newly developed interactive web server for analyzing the RNA sequencing expression data of 9,736 tumors and 8,587 normal samples from The Cancer Genome Atlas (TCGA) and the GTEx projects using a standard processing pipeline. It is able to perform survival and correlation analyses of DEGs (22). In the present study, candidate key genes were incorporated into the GEPIA database to further verify their expression in normal pancreatic and pancreatic cancer tissues. To produce the survival curves for key genes, the genes to be analyzed were searched in the main interface of the GEPIA database (http://gepia.cancer-pku. cn/index.html). Subsequently, 'Survival Plots' was selected in the analysis toolbar, the tumor type was set to PAAD and the confidence interval was set to $95 \%$. For the other parameters, the database's default settings were used.

Screening of cell lines for expression of ITGb6 by reverse transcription-quantitative $(R T-q) P C R$. A total of six pancreatic cancer cell lines (BXPC-3, CFPAC-1, MIA PaCa-2, ASPC-1, PANC-1 and SW1990) were provided by Shanghai GeneChem Co., Ltd. and cultured in RPMI-1640 basic medium (Corning, Inc.). All cells were routinely subcultured at $37^{\circ} \mathrm{C}$ in the presence of $5 \% \mathrm{CO}_{2}$ in an incubator with saturated humidity. Total RNA was extracted from the six cell lines using TRIzol reagent according to the manufacturer's instructions. RNA was reverse transcribed to complementary DNA using Promega M-MLV at $42^{\circ} \mathrm{C}$ (Promega Corp.). The mRNA expression levels of the ITGB6 gene in different cell lines of interest were detected by quantitative PCR using a LightCycler 480 II (Roche Molecular Systems, Inc.).The composition of the reaction mixture was SYBR premix ex taq $6.0 \mu 1$, primer mix $0.3 \mu 1$, reverse transcription product $0.6 \mu \mathrm{l}$ and RNase-free $\mathrm{H}_{2} \mathrm{O} 5.1 \mu \mathrm{l}$. The reaction conditions were as follows pre-denaturation at $95^{\circ} \mathrm{C}$ for $30 \mathrm{sec}$, followed by denaturation for $5 \mathrm{sec}$ at $95^{\circ} \mathrm{C}$ and annealing for $30 \mathrm{sec}$ at $60^{\circ} \mathrm{Cfor}$ a total of 40 cycles. The primer sequences were as follows: ITGB6 forward, 5'-TGATCT TCGCTGTAACCC-3' and reverse, 5'-CAGACCGCAGTT CTTCATA-3'; GAPDH forward, 5'-TGACTTCAACAGCGA CACCCA-3' and reverse, 5'-CACCCTGTTGCTGTAGCC AAA-3'. The experimental results were analyzed by the $2^{-\Delta \Delta \mathrm{Cq}}$ method (23) for relative quantitative analysis.

Lentivirus (LV) transfection to silence ITGB6 expression in the ASPC-1 cell line. Negative control (NC) virus CON077 and LV-ITGB6-RNA interference (RNAi) LV were constructed by Shanghai Jikai. The ITGB6-small interfering (si)RNA target sequence designed for the ITGB6 gene sequence was 5'-gcC TCCAAACATTCCCATGAT-3'. The CON077 sequence was 5'-TTCTCCGAACGTGTCACGT-3'. ASPC-1 cells with relatively high expression were selected for transfection and the following experimental groups were established: i) Mock group, normal ITGB6 cells; ii) Short hairpin (sh)NC group, ASPC-1 cells transfected with recombinant $\mathrm{LV}$ carrying NC-siRNA; iii) shITGB6 group, ASPC-1 cells transfected with recombinant LV carrying ITGB6-siRNA. Total RNA was extracted from cells in the shNC and shITGB6 groups using Total RNA extraction reagent (Shanghai Pufei) according to the manufacturer's instructions. and RT-qPCR was used to detect the expression levels of ITGB6 mRNA in the cells. 

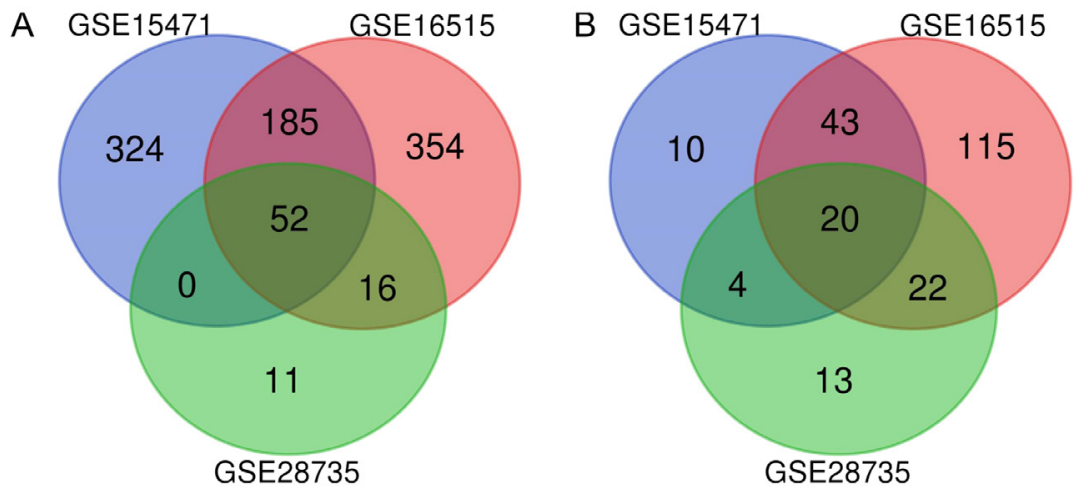

Figure 1. Analysis of the common differentially expressed genes in the microarray datasets of GSE28735, GSE16515 and GSE15471. (A) Upregulated genes. (B) Downregulated genes.

Cell proliferation assays. Cells transfected for $24 \mathrm{~h}$ were inoculated into 96-well plates and cultured for a further $24 \mathrm{~h}$ before MTT (Shanghai Dingguo Biotechnology Co., Ltd.) solution $(20 \mu \mathrm{l}$ at $5 \mathrm{~g} / \mathrm{l})$ was added to each well. After incubation at $37^{\circ} \mathrm{C}$ for $4 \mathrm{~h}$, the supernatant was discarded and dimethyl sulfoxide was added to each well (100 $\mu \mathrm{l})$. The absorbance of each well was determined at a wavelength of $490 \mathrm{~nm}$.

Cell cycle detection. The cells from the different experimental groups were digested with trypsin and centrifuged to collect the cells. After washing with D-Hanks' solution ( $\mathrm{pH} 7.2)$ pre-cooled at $4^{\circ} \mathrm{C}$, cells were fixed with $75 \%$ ethanol at $4{ }^{\circ} \mathrm{C}$ for $1 \mathrm{~h}$. The cell cycle was detected by flow cytometry, as previously described (24).

Statistical analysis. Values are expressed as the mean \pm standard deviation and all experiments were repeated independently three times. Statistical analyses were performed with SPSS 24.0 statistical software (IBM Corp.). Graphs and curves were constructed by GraphPad Prism 7 (GraphPad Software, Inc.). An independent-samples t-test was used to assess differences between paired samples. One-way ANOVA was used for comparison between groups with the least-significant difference method used for pairwise comparisons. $\mathrm{P}<0.05$ was considered to indicate a statistically significant difference.

\section{Results}

Identification of DEGs in PAAD. In the present study, the GEO2R analysis platform was used to preprocess and filter the original data of three selected datasets (GSE28735, GSE16515 and GSE15471). The Venn diagram (Fig. 1) contained a total of 72 genes with an intersection in the three datasets, including 20 downregulated and 52 upregulated genes (Table I).

Differential gene enrichment analysis. In order to further study the role of DEGs in the development of pancreatic cancer, Cluster Profiler was used to subject the DEGs to GO functional enrichment and KEGG pathway analysis. GO analysis indicated that the DEGs mainly act on the extracellular matrix (ECM); are involved in BPs such as cell-substrate adhesion, extracellular structure organization and ECM organization; and participate in the composition of the collagen-containing ECM and endoplasmic reticulum lumen. Other MFs such as ECM structural constituent, endopeptidase activity and glycosaminoglycan binding were also determined (Table II; Fig. 2A-C). Through KEGG signaling pathway analysis, it was indicated that the DEGs are mainly involved in human papillomavirus infection, ECM-receptor interaction, focal adhesion, PI3K-Akt signaling pathway, protein digestion and absorption, and pathways in pancreatic secretion (Table III; Fig. 2D).

Protein network and sub-network module analysis. A protein-protein interaction (PPI) network for DEGs was constructed using the STRING database (Fig. 3A). The first 10 nodes [fibronectin (FN)1, EGF, albumin (ALB), collagen $\alpha 1$ chain 1 (COL1A1), integrin subunit $\alpha 2$ (ITGA2), keratin 19(KRT19), collagen type XI $\alpha 1$ chain (COL11A1), thrombospondin 2 (THBS2), integrin subunit $\beta 6$ (ITGB6) and matrix metallopeptidase 12 (MMP12)] with the highest degrees were screened as hub genes. In order to further explore the association in the PPI network, the first three modules in the PPI network were extracted and certain key genes [periostin (POSTN), matrix metallopeptidase 11 (MMP11) and KRT19] were indicated to have local regulatory roles in the development and progression of pancreatic cancer (Fig. 3B). This provides additional grounds for studying the molecular mechanisms of pancreatic cancer.

Online survival analysis through GEPIA. To explore the association between the expression of key genes and the prognosis of patients with pancreatic cancer, the key genes were inputted into the GEPIA database for survival analysis. It was indicated that the expression levels of the ITGB6 and ITGA2 genes are closely associated with the patients' survival rate (Fig. 4A). This means that these genes have a negative impact on the survival time of patients with pancreatic cancer. Finally, using the GEPIA online database, it was verified that these genes were highly expressed in pancreatic cancer (Fig. 4B).

Expression of ITGB6 in various cell lines. As indicated by the RT-qPCR results, the expression levels of the gene ITGB6 were low in MIA PaCa-2 and PANC-1 cells, but high in BXPC-3, CFPAC-1, ASPC-1 and SW1990 cells (Fig. 5A). Therefore, ASPC-1 cells were selected for the subsequent experiments.

Results of LV-ITGB6-RNAi lentivirus infection in AsPC-1 cells. Observation of the green fluorescent protein encoded 
Table I. Differentially expressed genes $(n=72)$.

Item Gene names

Downregulated genes

ALB, ANPEP, AQP8, CELA2B, EGF, ERO1B, ERP27, FGL1, GP2, KIAA1324, KLK1, PAIP2B, PDIA2, PDK4, PNLIPRP1, PNLIPRP2, RBPJL, SERPINI2, TMED6, TRHDE

Upregulated genes

AGR2, AHNAK2, ANLN, ANTXR1, ANXA10, CEACAM5, CEACAM6, CEMIP, CLDN18, COL10A1, COL11A1, COL12A1, COL1A1, CP, CST1, CTRL, CTSE, CXCL5, DPCR1, EDIL3, FERMT1, FN1, FXYD3, GABRP, GATM, INHBA, ITGA2, ITGB6, KRT17, KRT19, KRT7, LAMB3, LAMC2, MMP11, MMP12, NOX4, NR5A2, PLAC8, POSTN, SDR16C5, SERPINB5, SLC6A14, SLPI, SULF1, TCN1, TFF1, THBS2, TMC5, TMPRSS4, TSPAN1, VCAN, VSIG1
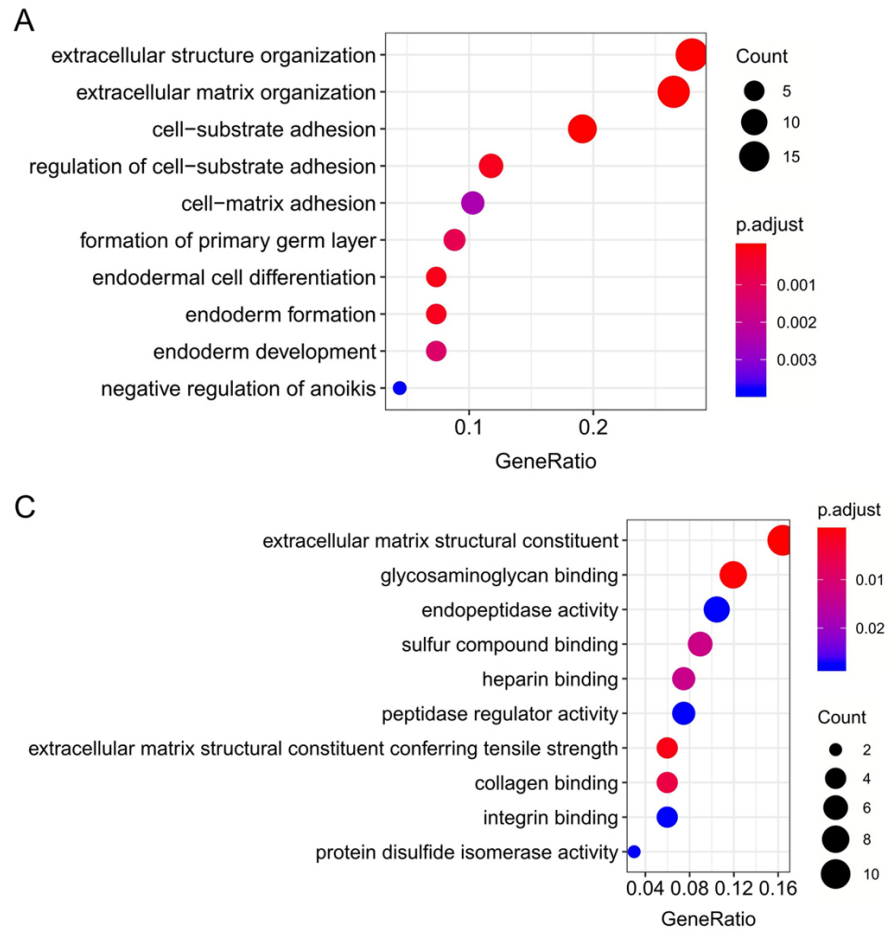

B
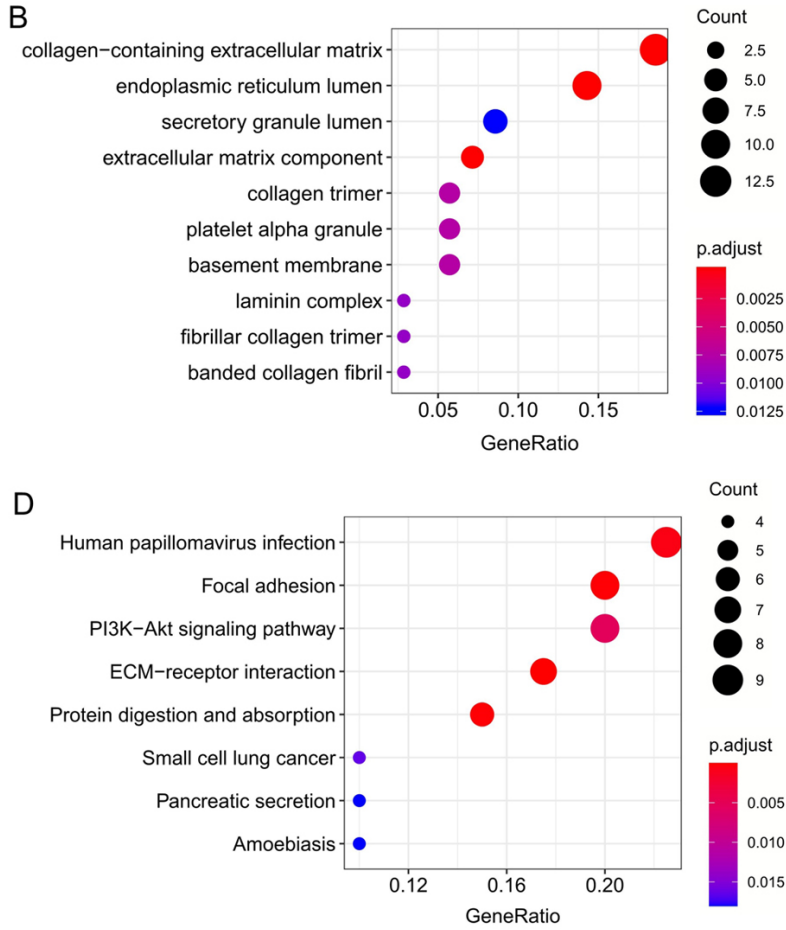

Figure 2. Functional enrichment and pathway analysis. (A-C) Gene ontology terms in the categories (A) biological process, (B) cellular component and (C) molecular function. (D) Kyoto Encyclopedia of Genes and Genomes pathways. ECM, extracellular matrix.

in the plasmid with ITGB6 under a fluorescence microscope confirmed that $24 \mathrm{~h}$ after AsPC-1 cells had been infected with the recombinant virus LV-ITGB6-RNAi, the expression of green fluorescent protein was observed in both groups of cells (Fig. 5B). The transfection efficiency detected by flow cytometry was $>80 \%$. RT-qPCR analysis demonstrated that the ITGB6 silencing effect of the plasmid was evident $72 \mathrm{~h}$ after infection $(\mathrm{P}<0.05$; Fig. 5C).

Effect of ITGB6 on the proliferation of pancreatic cancer ASPC-1 cells. According to the results of the growth curve analysis, compared with that of the shNC group, the growth curve of the shITGB6 group was flatter and the overall growth rate of cells was significantly reduced. From the fourth day onwards, the proliferation rate of cells in the experimental group was significantly lower than that of cells in the control group and the difference was statistically significant $(\mathrm{P}<0.01)$. It was indicated that knockdown of ITGB6 significantly inhibited the proliferation of ASPC-1 cells (Fig. 6A).
Effect of ITGB6 on the cell cycle of pancreatic cancer ASPC-1 cells. Flow cytometric analysis of the cell cycle with PI indicated that, compared with that of the shNC group, the percentage of cells in G1- and S-phase in the shITGB6 group was significantly reduced $(\mathrm{P}<0.05)$, while the percentage of cells in $\mathrm{G} 2 / \mathrm{M}$-phase was significantly increased $(\mathrm{P}<0.01)$. This suggested that silencing of ITGB6 gene expression causes cell-cycle arrest in the G2/M phase, thus significantly inhibiting the cell cycle (Fig. 6B and C).

\section{Discussion}

Pancreatic cancer is a highly malignant tumor type of the digestive tract (25). Due to the lack of specific clinical manifestations in the early stage of the disease, $>80 \%$ of patients are diagnosed at an advanced stage, where treatment becomes markedly difficult (26). Revealing the molecular mechanisms involved in the development of pancreatic cancer will help to discover novel tumor markers that may allow for an early diagnosis of 
A

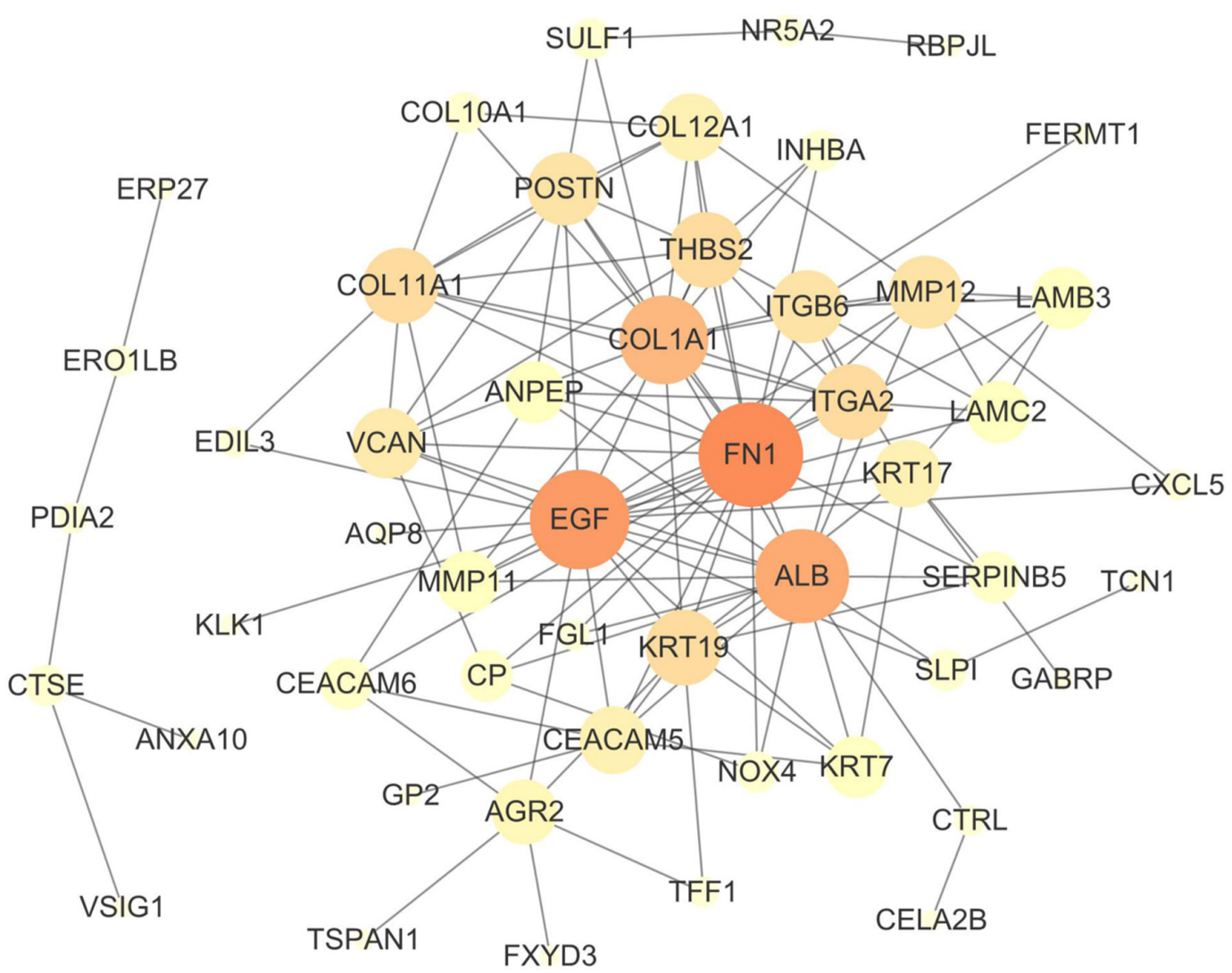

B
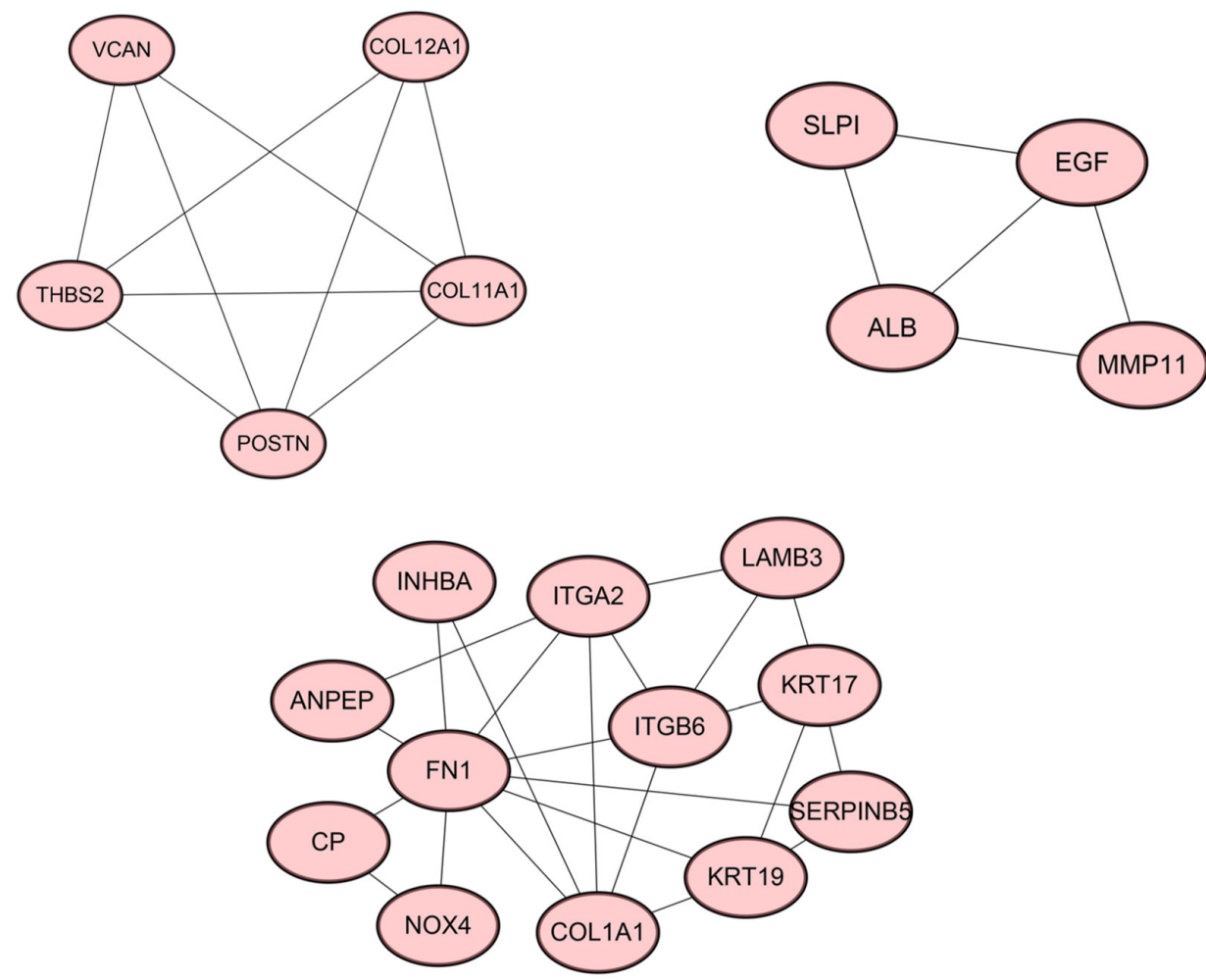

Figure 3. PPI network complex of DEGs in pancreatic adenocarcinoma. (A) PPI networks of the DEGs. In the figure, a larger sphere area indicates a greater degree value of the target protein. Lines represent the protein interaction between the genes. (B) Three sub-modules in a PPI network. POSTN, MMP11 and KRT19 are located in three sub-modules. PPI, protein-protein interaction; DEG, differentially expressed gene; POSTN, periostin; MMP11, matrix metallopeptidase 11; KRT19, keratin 19.

pancreatic cancer, develop novel effective treatment strategies, evaluate prognosis and improve patient survival. In the present study, datasets of pancreatic cancer-related chips from the GEO database were analyzed and 72 significant DEGs were mined. 
A
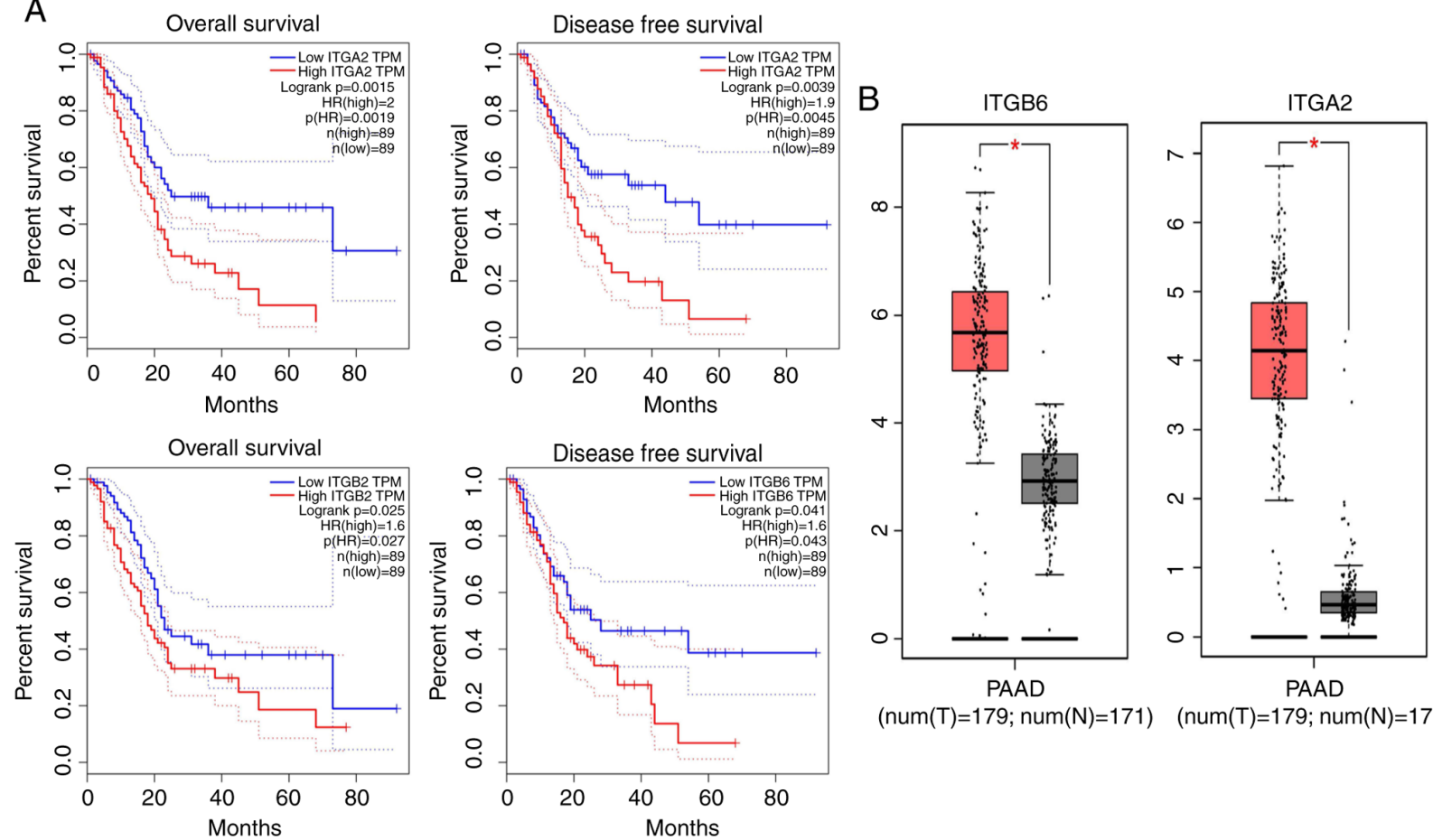

$(\operatorname{num}(T)=179 ; \operatorname{num}(N)=171) \quad(\operatorname{num}(T)=179 ; \operatorname{num}(N)=171)$

Figure 4. Prognostic values of hub genes in patients with PAAD from the GEPIA database. (A) Survival analysis of hub genes in protein-protein interaction networks (overall survival and disease-free survival for hub genes). Dotted lines indicate the $95 \%$ confidence interval range. Red represents high gene expression, blue represents low gene expression. (B) Verification results of key gene expression in pancreatic cancer tissues. Red color indicates tumor tissues, while grey color indicates normal tissues. Each dot representing a distinct tumor or normal sample. "P<0.05. PAAD, pancreatic adenocarcinoma; HR, hazard ratio; ITGA2, integrin subunit $\alpha 2$; T, tumor sample; N, normal sample; TPM, Transcripts Per Kilobase of exon model per Million mapped reads.

A

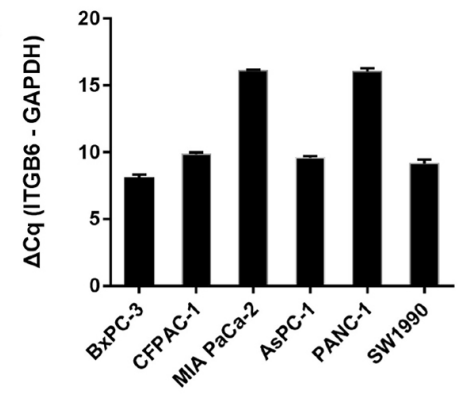

Expression abundance of ITGB6 gene in different cell lines

C

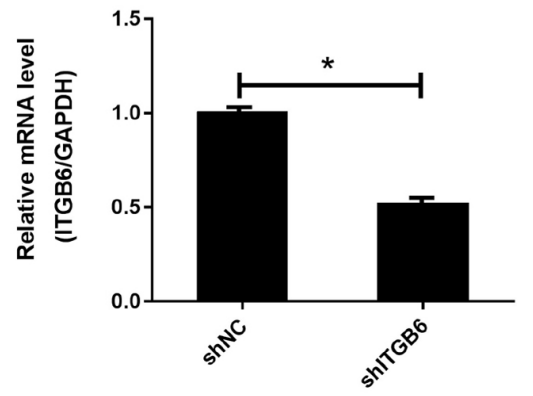

B
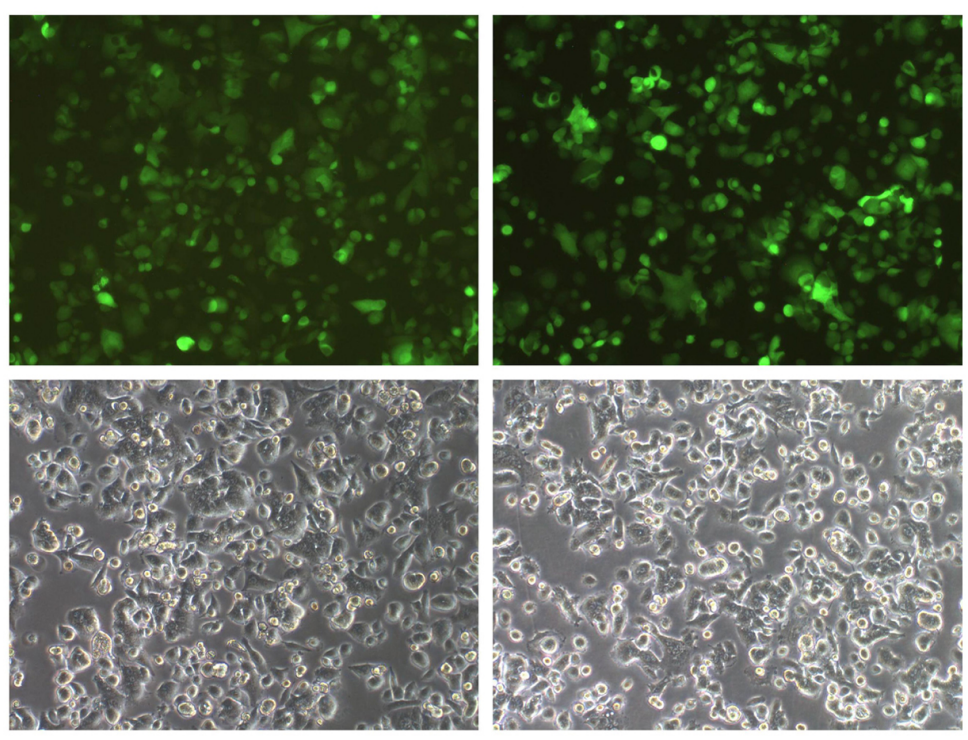

shNC

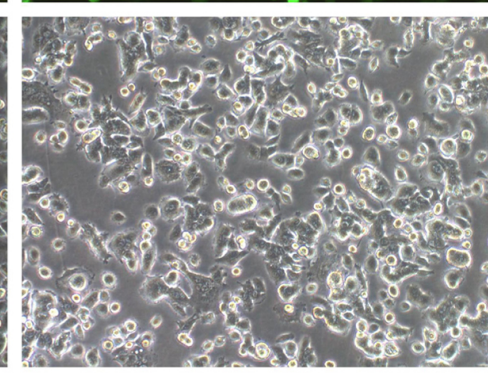

shITGB6

Figure 5. (A) Expression abundance of the ITGB6 gene in different cell lines. When the $\Delta$ Cq value was $\leq 12$, the gene expression abundance in the cell was high; when the $\Delta \mathrm{Cq}$ value was $\geq 16$, the gene expression abundance in the cell was low. (B) Recombinant lentivirus infection image in ASPC-1 cells (upper panels, bright field; lower panels, green fluorescence field; magnification, x100). (C) Results of the reverse transcription-quantitative PCR analysis of ITGB6. ${ }^{*}<0.05$. ITGA6, integrin subunit $\beta$ 6; shNC, negative control shRNA; shITGB6, shRNA targeting ITGB6; shRNA, short hairpin RNA; Cq, quantification cycle.

GO functional analysis indicated that the DEGs are mainly involved in BPs such as cell adhesion and ECM decomposition.
KEGG signaling pathway analysis revealed the involvement of focal adhesion, ECM-receptor interaction, PI3K-Akt signaling 

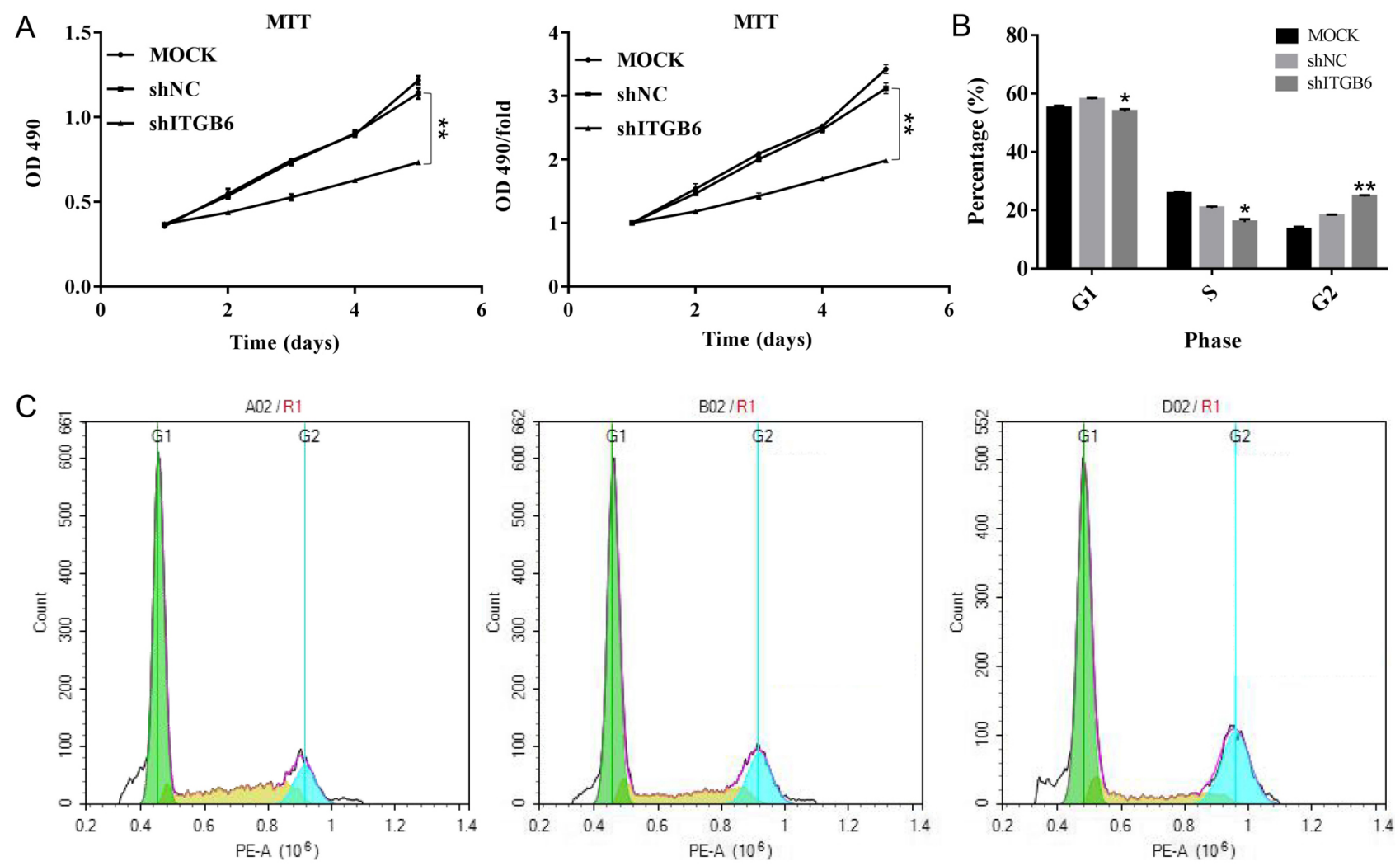

Figure 6. (A) Effect of ITGB6 gene silencing on the proliferation of pancreatic cancer ASPC-1 cells. The OD490 value indicates the number of viable cells, while the OD490/fold indicates the multiplication ratio on each day. (B and C) Effect of ITGB6 gene silencing on the cell cycle of pancreatic cancer ASPC-1 cells. Compared with the number of cells in each phase of the cell cycle in the control group, the results of the statistical analysis of the number of cells in G1, $\mathrm{S}$ and $\mathrm{G} 2$ phases in shITGB6 group was $\mathrm{P}<0.05$. ${ }^{*} \mathrm{P}<0.05,{ }^{* *} \mathrm{P}<0.01$. OD490, optical density at $490 \mathrm{~nm}$; ITGB6, integrin subunit $\beta$ 6; shNC, negative control shRNA; shITGB6, shRNA targeting ITGB6; shRNA, short hairpin RNA.

pathway and protein digestion and absorption. Of these, the PI3K-Akt signaling pathway is particularly noteworthy, as it is known to be an important oncogenic signaling pathway involved in tumorigenesis and resistance to targeted anticancer therapies for various tumor types (27). The PI3K-Akt signaling pathway is also an important cause of pancreatic cancer and is associated with poor prognosis of pancreatic cancer. Abnormal activation of this pathway involves cellular metabolism and survival and cell cycle progression. Several inhibitors targeting Akt, PI3K and mTOR have recently been developed for clinical research (28).

In the present study, the STRING online tool was used to analyze the PPIs encoded by the DEGs associated with pancreatic cancer. It was determined that the interactions between the proteins encoded by these genes were mainly concentrated in key node genes such as FN1, EGF, ALB, ITGA2 and ITGB6. FN1 exhibited the highest connectivity in the PPI network, suggesting its important role in pancreatic cancer. A recent study (29) revealed that FN1 was abundantly expressed in the tumor microenvironment of PAAD, which is consistent with the present study, demonstrating that FN1 is highly expressed in PAAD, while its expression in normal pancreatic tissue was low or marginal. Expression of FN1 matrix was associated with aggressive tumor characteristics, including greater tumor size and advanced $\mathrm{T}$ and $\mathrm{N}$ stages.

ALB and EGF were the only downregulated genes among the hub genes. The ALB gene, which encodes the most abundant protein in human blood, was a downregulated gene in pancreatic cancer. The protein regulates plasma colloid osmotic pressure and serves as a carrier protein for a variety of endogenous molecules and exogenous drugs. A recent study indicated that the combined detection of the derived neutrophil-to-lymphocyte ratio and ALB are able to improve the diagnostic efficiency for pancreatic cancer (30). Another study demonstrated the C-reactive protein (CRP)/ALB ratio may serve as a significant and promising inflammatory prognostic score in pancreatic cancer, since high CRP/Alb indicates a poor prognosis (31). Combined with the results of the present study, this suggests that the detection of ALB levels in patients with pancreatic cancer may improve the sensitivity of pancreatic cancer diagnosis. EGF was indicated to be significantly downregulated in the present study, which is inconsistent with the results of Hao et al (32). A study with a larger sample of patients is required for further validation.

Furthermore, the present study detected novel genes involved in the local regulation of the development of pancreatic cancer, including POSTN, MMP11 and KRT19. PPI module analysis indicated the important role of these central genes, which are involved in key pathways and BPs associated with pancreatic cancer.

COL1A1 is a key structural component of the ECM. It occurs in the majority of connective and embryonic tissues, and is an important member of the collagen family (33). Typically, type I collagen consists of COL1A1 and COL1A2 (34). Abnormal expression of COL1A1 has been reported in kidney cancer, hepatocellular carcinoma and melanoma (35-37). Li et al (38) 
Table II. GO enrichment analysis of the differentially expressed genes.

\begin{tabular}{llrll}
\hline Category & \multicolumn{1}{c}{ Term } & Count & P-value & Exemplary genes \\
\hline BP & GO:0030198 extracellular matrix organization & 12 & $3.55 \times 10^{-10}$ & LAMB3, ERO1B, ITGB6 \\
BP & GO:0007155 cell adhesion & 12 & $2.12 \times 10^{-6}$ & LAMB3, ITGB6, FERMT1 \\
BP & GO:0035987 endodermal cell differentiation & 5 & $4.02 \times 10^{-6}$ & INHBA, LAMB3, COL12A1 \\
BP & GO:0030574 collagen catabolic process & 6 & $5.94 \times 10^{-6}$ & COL1A1, MMP12, COL11A1 \\
BP & GO:0022617 extracellular matrix disassembly & 5 & $2.53 \times 10^{-4}$ & LAMB3, LAMC2, FN1 \\
CC & GO:0005615 extracellular space & 24 & $4.85 \times 10^{-10}$ & CXCL5, CST1, POSTN \\
CC & GO:0005576 extracellular region & 22 & $3.63 \times 10^{-7}$ & PNLIPRP1, PNLIPRP2, CXCL5 \\
CC & GO:0070062 extracellular exosome & 27 & $7.40 \times 10^{-6}$ & FXYD3, TSPAN1, KIAA1324 \\
CC & GO:0031012 extracellular matrix & 9 & $1.74 \times 10^{-5}$ & COL12A1, SLPI, POSTN \\
MF & GO:0005509 calciumion binding & 10 & 0.001453 & PNLIPRP1, SULF1, ANXA10 \\
MF & GO:0004252 serine-type endopeptidase activity & 6 & 0.002791 & CELA2B, KLK1, MMP12 \\
MF & GO:0003756 protein disulfide isomerase activity & 3 & 0.003112 & ERO1B, ERP27, PDIA2 \\
MF & GO:0005178 integrin binding & 4 & 0.007401 & ITGB6, ITGA2, EDIL3, FN1 \\
\hline
\end{tabular}

GO, gene ontology; BP, biological process; CC, cellular component; MF, molecular function.

Table III. Kyoto Encyclopedia of Genes and Genomes pathways significantly enriched by the differentially expressed genes.

\begin{tabular}{lccc}
\hline Term & Count & P-value & Exemplary genes \\
\hline hsa04512:ECM-receptor interaction & 8 & $1.06 \times 10^{-7}$ & LAMB3, ITGB6, ITGA2 \\
hsa04510:Focal adhesion & 9 & $3.20 \times 10^{-6}$ & LAMB3, ITGB6, ITGA2 \\
hsa04151:PI3K-Akt signaling pathway & 6 & $1.34 \times 10^{-4}$ & LAMB3, ITGB6, ITGA2 \\
hsa04974:Protein digestion and absorption & 9 & $4.69 \times 10^{-5}$ & COL12A1, CELA2B, COL1A1 \\
hsa04972:Pancreatic secretion & 4 & 0.00893 & PNLIPRP2, CELA2B, CTRL \\
\hline
\end{tabular}

ECM, extracellular matrix; Hsa, Homo sapiens.

indicated that COL1A1 and COL1A2 were overexpressed in gastric cancer and high COL1A1 may be a monitoring factor for early gastric cancer or a prognostic factor for predicting overall survival. COL1A1 secreted by pancreatic stellate cells (PSCs) promotes invasion and migration of pancreatic cancer cells (39). A previous study (40) has indicated that inhibition of COL1A1 with the novel hydrophilic agent palmatine (PMT) is able to inhibit the growth of PSCs. The role of collagen family members in pancreatic cancer deserves further investigation.

Improving the prognosis of patients with pancreatic cancer is an urgent problem to be solved. In the present study, the key genes selected from pancreatic cancer samples in TCGA database were analyzed and the results suggested that high expression of the ITGA2 and ITGB6 genes is a high-risk factor for poor prognosis in patients with pancreatic cancer. This evaluation of prognosis has obvious clinical significance and future studies focusing on these genes may contribute to the treatment of pancreatic cancer. ITGA2 is an essential collagen receptor on epithelial cells. Gong et al (41) suggested that changes in UCA1 (urothelial cancer associated 1) Expression may affect the expression of ITGA2, further interfering with the progression of cancer. The adhesion spot pathway was identified as the regulatory mechanism of ITGA2. The expression profile of the long non-coding RNA UCA1 was associated with the migration and apoptosis of SW-1990 cells (42). Specifically, upregulated co-expression of UCA1-ITGA2 promoted the migration and invasion of pancreatic cancer cells. Thus, ITGA2 may become a novel potential target for gene therapy.

The mechanisms of ITGB6 in cancer have remained largely elusive and studying the TGF- $\beta$ /ITGB6 signaling pathway may be worthwhile. ITGB6 has a role in signal transmission from the ECM to cells. TGF- $\beta$ is an important inflammatory factor produced by macrophages, stromal cells and tumor cells in the tumor microenvironment and is involved in the occurrence, development and metastasis of tumors. A previous study (43) indicated that the TGF- $\beta$ /ITGB6 signaling pathway has an important role in the invasion and metastasis of esophageal squamous cell carcinoma and the present study suggested that this pathway may be inhibited by miR-17/20a. A recent bioinformatics study on pancreatic cancer used ITGB6 as an independent risk factor for the prognosis of pancreatic cancer, which also supports the present results (44). However, the function and mechanism of ITGB6 in pancreatic cancer are unclear and further studies should be performed to detect the levels of this gene and confirm its role in PAAD. The present study suggested that the expression of ITGB6 mRNA was 
high in the majority of pancreatic cancer cell lines evaluated, including BXPC-3, CFPAC-1, ASPC-1 and SW1990. On the contrary, according to the RT-qPCR results, the expression of ITGB6 in the MIA PaCa-2 and PANC-1 cell lines was lower than that in the other four cell lines. Infinite cell proliferation caused by imbalances in various stages of the cell cycle is closely associated with formation of tumors (45). In the cellular functional experiments of the present study, the role of ITGB6 as an oncogene was confirmed. Through functional analyses with silencing of ITGB6 and determination of the cell proliferation and cell cycle distribution in ASPC-1 cells, it was indicated that inhibition of ITGB6 decreased cell proliferation and induced $\mathrm{G} 2 / \mathrm{M}$ arrest, which supports the results of the present bioinformatics analysis. The effect of silencing the ITGB6 gene on the cell cycle may be a potential mechanism for inhibition of further progression of pancreatic tumors. The above results strongly supported the possibility of ITGB6 as an optimal target for therapeutic intervention. Detection of the cell cycle, particularly the expression of $\mathrm{G} 2 / \mathrm{M}$ phase-related regulatory proteins, will be the focus of future studies by our group. At the same time, the study still has certain limitations, such as the lack of western blot data. Further research on the impact of ITGB6 on pancreatic cancer cell invasion and migration, and the potential molecular mechanism of ITGB6 in pancreatic cancer, require to be further explored.

In conclusion, the present study employed a series of bioinformatics methods to identify key genes in pancreatic cancer. ITGA 2 and ITGB6 may be used as potential biomarkers for the diagnosis and treatment of patients with pancreatic cancer. The DEGs and metabolic pathways revealed in the present study may help us understand the mechanisms of the molecular development of pancreatic cancer and provide a theoretical basis for future research on clinical targeted therapies. Analytical data mining through bioinformatics analysis is a feasible method to systematically identify potential biomarkers. However, the molecular mechanisms of pancreatic cancer require to be further investigated through biological experiments.

\section{Acknowledgements}

Not applicable.

\section{Funding}

The present study was supported by Zhenjiang Science and Technology Committee (grant. no. SH 2019061).

\section{Availability of data and materials}

The datasets used and/or analyzed during the present study are available from the corresponding author on reasonable request.

\section{Authors' contributions}

XX and TR were responsible for designing the study, performing the experiment, collecting the data and writing the manuscript. XW, XZ and SD were responsible for designing the study, performing the experiment, and collecting the data and reviewing the manuscript. SD was responsible for providing experimental ideas and reviewing the manuscript. $\mathrm{XX}$ and TR were responsible for the confirming the authenticity of all the raw data. All authors read and approved the final manuscript.

\section{Ethics approval and consent to participate}

All animal experimentation protocols were approved by the Institutional Animal Care and Use Committees of Jiangsu University and were conducted according to the Regulation on Animal Experimentation at Jiangsu University.

\section{Patient consent for publication}

Not applicable.

\section{Competing interests}

The authors declare that they have no competing interests.

\section{References}

1. Rizzato C, Campa D, Talar-Wojnarowska R, Halloran C, Kupcinskas J, Butturini G, Mohelníková-Duchoňová B, Sperti C, Tjaden C, Ghaneh P, et al: Association of genetic polymorphisms with survival of pancreatic ductal adenocarcinoma patients. Carcinogenesis 37: 957-964, 2016

2. Vincent A, Herman J, Schulick R, Hruban RH and Goggins M: Pancreatic cancer. Lancet 378: 607-620, 2011.

3. Rahib L, Smith BD, Aizenberg R, Rosenzweig AB, Fleshman JM and Matrisian LM: Projecting cancer incidence and deaths to 2030: The unexpected burden of thyroid, liver, and pancreas cancers in the United States. Cancer Res 74: 2913-2921, 2014.

4. Halbrook CJ and Lyssiotis CA: Employing metabolism to improve the diagnosis and treatment of pancreatic cancer. Cancer Cell 31: 5-19, 2017

5. Griffin JF, Poruk KE and Wolfgang CL: Pancreatic cancer surgery: Past, present, and future. Chin J Cancer Res 27: 332-348, 2015.

6. Maitra A, Kern SE and Hruban RH: Molecular pathogenesis of pancreatic cancer. Best Pract Res Clin Gastroenterol 20: 211-226, 2006.

7. Long J, Liu Z, Wu X, Xu Y and Ge C: Gene expression profile analysis of pancreatic cancer based on microarray data. Mol Med Rep 13: 3913-3919, 2016.

8. Morris JP IV, Wang SC and Hebrok M: KRAS, hedgehog, wnt and the twisted developmental biology of pancreatic ductal adenocarcinoma. Nat Rev Cancer 10: 683-695, 2010.

9. Lauth $\mathrm{M}$ and Toftgård R: Hedgehog signaling and pancreatic tumor development. Adv Cancer Res 110: 1-17, 2011.

10. Shigeyasu K, Toden S, Zumwalt TJ, Okugawa Y and Goel A: Emerging role of MicroRNAs as liquid biopsy biomarkers in gastrointestinal cancers. Clin Cancer Res 23: 2391-2399, 2017.

11. Cui Z, Liu G and Kong D: miRNA27a promotes the proliferation and inhibits apoptosis of human pancreatic cancer cells by wnt/ $\beta$-catenin pathway. Oncol Rep 39: 755-763, 2018.

12. Zhao DW, Hou YS, Sun FB, Han B and Li SJ: Effects of miR-132 on proliferation and apoptosis of pancreatic cancer cells via hedgehog signaling pathway. Eur Rev Med Pharmacol Sci 23: 1978-1985, 2019.

13. Barrett T, Wilhite SE, Ledoux P, Evangelista C, Kim IF, Tomashevsky M, Marshall KA, Phillippy KH, Sherman PM, Holko M, et al: NCBI GEO: Archive for functional genomics data sets-update. Nucleic Acids Res 41: D991-D995, 2013.

14. Clough E and Barrett T: The gene expression omnibus database. Methods Mol Biol 1418: 93-110, 2016.

15. Zhang G, Schetter A, He P, Funamizu N, Gaedcke J, Ghadimi BM, Ried T, Hassan R, Yfantis HG, Lee DH, et al: DPEP1 inhibits tumor cell invasiveness, enhances chemosensitivity and predicts clinical outcome in pancreatic ductal adenocarcinoma. PLoS One 7: e31507, 2012.

16. Pei H, Li L, Fridley BL, Jenkins GD, Kalari KR, Lingle W, Petersen G, Lou Z and Wang L: FKBP51 affects cancer cell response to chemotherapy by negatively regulating Akt. Cancer Cell 16: 259-266, 2009. 
17. Badea L, Herlea V, Dima SO, Dumitrascu T and Popescu I: Combined gene expression analysis of whole-tissue and microdissected pancreatic ductal adenocarcinoma identifies genes specifically overexpressed in tumor epithelia. Hepatogastroenterology 55: 2016-2027, 2008.

18. Yu G, Wang LG, Han Y and He QY: clusterProfiler: An R package for comparing biological themes among gene clusters. OMICS 16: 284-287, 2012.

19. Szklarczyk D, Franceschini A, Wyder S, Forslund K, Heller D, Huerta-Cepas J, Simonovic M, Roth A, Santos A, Tsafou KP, et al: STRING v10: Protein-protein interaction networks, integrated over the tree of life. Nucleic Acids Res 43: D447-D452, 2015.

20. Gene Ontology Consortium: The gene ontology (GO) project in 2006. Nucleic Acids Res 34: D322-D326, 2006.

21. Ashburner M, Ball CA, Blake JA, Botstein D, Butler H, Cherry JM, Davis AP, Dolinski K, Dwight SS, Eppig JT, et al: Gene ontology: Tool for the unification of biology. The gene ontology consortium. Nat Genet 25: 25-29, 2000.

22. Tang Z, Li C, Kang B, Gao G, Li C and Zhang Z: GEPIA: A web server for cancer and normal gene expression profiling and interactive analyses. Nucleic Acids Res 45: W98-W102, 2017.

23. Livak KJ and Schmittgen TD: Analysis of relative gene expression data using real-time quantitative PCR and the 2(-Delta Delta C(T) method. Methods 25: 402-408, 2001.

24. Krishan A: Rapid flow cytofluorometric analysis of mammalian cell cycle by propidium iodide staining. J Cell Biol 66: 188-193, 1975.

25. Ilic M and Ilic I: Epidemiology of pancreatic cancer. World J Gastroenterology 22: 9694-9705, 2016.

26. Miller KD, Siegel RL, Lin CC, Mariotto AB, Kramer JL, Rowland JH, Stein KD, Alteri R and Jemal A: Cancer treatment and survivorship statistics, 2016. CA Cancer J Clin 66: 271-289, 2016.

27. Courtney KD, Corcoran RB and Engelman JA: The PI3K pathway as drug target in human cancer. J Clin Oncol 28: 1075-1083, 2010

28. Ebrahimi S, Hosseini M, Shahidsales S, Maftouh M, Ferns GA, Ghayour-Mobarhan M, Hassanian SM and Avan A: Targeting the Akt/PI3K signaling pathway as a potential therapeutic strategy for the treatment of pancreatic cancer. Curr Med Chem 24: $1321-1331,2017$

29. Hu D, Ansari D, Zhou Q, Sasor A, Hilmersson KS and Andersson R: Stromal fibronectin expression in patients with resected pancreatic ductal adenocarcinoma. World J Surg Oncol 17: 29, 2019.

30. Liu JX, Li A, Zhou LY, Liu XF, Wei ZH, Wang XZ and Ying HQ: Significance of combined preoperative serum Alb and dNLR for diagnosis of pancreatic cancer. Future Oncol 14: 229-239, 2018.

31. Liu Z, Jin K, Guo M, Long J, Liu L, Liu C, Xu J, Ni Q, Luo G and $\mathrm{Yu} \mathrm{X}$ : Prognostic value of the CRP/Alb ratio, a novel inflammation-based score in pancreatic cancer. Ann Surg Oncol 24: 561-568, 2017.

32. Hao C, Li Z, Zhang X, Zhang H, Shang H, Bao J and Wang H: Expression and clinical significance of EGF and TGF-alpha in chronic pancreatitis and pancreatic cancer. Minerva Endocrinol 43: 253-258, 2018.
33. Cole WG: Collagen genes: Mutations affecting collagen structure and expression. Prog Nucleic Acid Res Mol Biol 47: 29-80, 1994.

34. Exposito JY, Valcourt U, Cluzel C and Lethias C: The fibrillar collagen family. Int J Mol Sci 11: 407-426, 2010.

35. de Caceres II, Dulaimi E, Hoffman AM, Al-Saleem T, Uzzo RG and Cairns P: Identification of novel target genes by an epigenetic reactivation screen of renal cancer. Cancer Res 66: 5021-5028, 2006.

36. Hayashi M, Nomoto S, Hishida M, Inokawa Y, Kanda M, Okamura Y, Nishikawa Y, Tanaka C, Kobayashi D, Yamada S, et al: Identification of the collagen type $1 \alpha 1$ gene (COL1A1) as a candidate survival-related factor associated with hepatocellular carcinoma. BMC Cancer 14: 108, 2014.

37. Bonazzi VF, Nancarrow DJ, Stark MS, Moser RJ, Boyle GM, Aoude LG, Schmidt C and Hayward NK: Cross-platform array screening identifies COL1A2, THBS1, TNFRSF10D and UCHL1 as genes frequently silenced by methylation in melanoma. PLoS One 6: e26121, 2011.

38. Li J, Ding Y and Li A: Identification of COL1A1 and COL1A2 as candidate prognostic factors in gastric cancer. World J Surg Oncol 14: 297, 2016.

39. Ikenaga N, Ohuchida K, Mizumoto K, Akagawa S, Fujiwara K, Eguchi D, Kozono S, Ohtsuka T, Takahata S and Tanaka M: Pancreatic cancer cells enhance the ability of collagen internalization during epithelial-mesenchymal transition. PLoS One 7: e40434, 2012.

40. Chakravarthy D, Muñoz AR, Su A, Hwang RF, Keppler BR, Chan DE, Halff G, Ghosh R and Kumar AP: Palmatine suppresses glutamine-mediated interaction between pancreatic cancer and stellate cells through simultaneous inhibition of survivin and COL1A1. Cancer Lett 419: 103-115, 2018.

41. Gong J, Lu X, Xu J, Xiong W, Zhang H and Yu X: Coexpression of UCA1 and ITGA2 in pancreatic cancer cells target the expression of miR-107 through focal adhesion pathway. J Cell Physiol 234: 12884-12896, 2018

42. Chen P, Wan D, Zheng D, Zheng Q, Wu F and Zhi Q: Long non-coding RNA UCA1 promotes the tumorigenesis in pancreatic cancer. Biomed Pharmacother 83: 1220-1226, 2016.

43. Jing C, Ma G, Li X, Wu X, Huang F, Liu K and Liu Z: MicroR NA-17/20a impedes migration and invasion via TGF-3/ITGB6 pathway in esophageal squamous cell carcinoma. Am J Cancer Res 6: 1549-1562, 2016.

44. Wu M, Li X, Zhang T, Liu Z and Zhao Y: Identification of a nine-gene signature and establishment of a prognostic nomogram predicting overall survival of pancreatic cancer. Front Oncol 9: 996, 2019.

45. Zhou Y, Chen Y, Ding W, Hua Z, Wang L, Zhu Y, Qian H and Dai T: LncRNA UCA1 impacts cell proliferation, invasion, and migration of pancreatic cancer through regulating miR-96/FOXO3. IUBMB life 70: 276-290, 2018.

This work is licensed under a Creative Commons Attribution-NonCommercial-NoDerivatives 4.0 International (CC BY-NC-ND 4.0) License. 